\title{
Synthesis, Biological Evaluation of Fluorescent 23-Hydroxybetulinic Acid Probes, and Their Cellular Localization Studies
}

\author{
Hong Yao, ${ }^{\dagger}$ Guoxiang Wei, ${ }^{\dagger}$ Yanpeng Liu, ${ }^{\dagger}$ Hequan Yao, ${ }^{\dagger}$ Zheying Zhu, ${ }^{\ddagger}$ Wencai Ye, ${ }^{\S}$ Xiaoming Wu, ${ }^{\dagger}$ \\ Jinyi $\mathrm{Xu}, *, \dagger$ and Shengtao $\mathrm{Xu}^{*, \dagger}$ \\ ${ }^{\dagger}$ Department of Medicinal Chemistry and State Key Laboratory of Natural Medicines, China Pharmaceutical University, 24 Tong Jia \\ Xiang, Nanjing 210009, China \\ ${ }^{\ddagger}$ Division of Molecular Therapeutics \& Formulation, School of Pharmacy, The University of Nottingham, University Park Campus, \\ Nottingham NG7 2RD, U.K. \\ ${ }^{\S}$ College of Pharmacy, Jinan University, Guangzhou 510632, China
}

Supporting Information

ABSTRACT: 23-Hydroxybetulinic acid (23-HBA) is a complex lupane triterpenoid, which has attracted increasing attention as an anticancer agent. However, its detailed mechanism of anticancer action remains elusive so far. To reveal its anticancer mode of action, a series of fluorescent 23HBA derivatives conjugated with coumarin dyes were designed, synthesized, and evaluated for their antiproliferative activities. Subcellular localization and uptake profile studies of representative fluorescent 23-HBA probe 26c were performed in $\mathrm{B} 16 \mathrm{~F} 10$ cells, and the results suggested that probe $26 \mathrm{c}$ was rapidly taken up into $\mathrm{B} 10 \mathrm{~F} 10$ cells in a dose-dependent manner and mitochondrion was the main site of its accumulation. Further mode of action studies implied that the mitochondrial pathway was involved in 23-HBA-mediated apoptosis. Together, our results provided new clues for revealing the molecular mechanism of natural product 23-HBA for its further development into an antitumor agent.

KEYWORDS: Betulinic acid, Fluorescent probe, Antitumor, Mechanism, Mitochondrion
$\mathrm{N}$ atural products represent an enormous source of pharmacologically active compounds and are often used as the starting point in modern drug discovery. ${ }^{1}$ 23Hydroxybetulinic acid (23-HBA, 1, Figure 1) is a natural

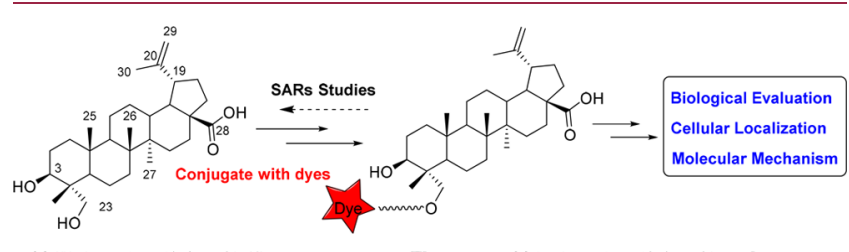

23-Hydroxybetulinic acid (1)

Fluorescent 23-hydroxybetulinic acid probe

Figure 1. Overall strategy of fluorescent 23-HBA probe for subcellular localization and mechanism studies.

lupane-type pentacyclic triterpene isolated from the root of the traditional Chinese herb Pulsatilla chinensis. ${ }^{2}$ In recent years, 23-HBA has been attracting considerable attention due to its remarkable antineoplastic activities. ${ }^{3-6}$ Several reports have indicated that the anticancer mechanisms of 23-HBA or its derivatives may be associated with depolarization of the mitochondrial membrane potential (MMP) and subsequent cell apoptosis. ${ }^{7,8}$ However, the detailed molecular mechanism and targets underlying the antitumor activities of 23-HBA are still not fully understood.

In recent years, much progress has been made in establishing methods for the identification of the cellular targets or mode of action (MOA) of small molecules, ${ }^{9}$ but current approaches mostly require lysis or rely on indirect assays. Ideal strategies that are capable of sensitive detection of biological processes in living systems (e.g., live cells, tissues, or animals) are required for the MOA studies and target proteins identification. ${ }^{10}$

Fluorescence-based cell-imaging strategies are highly useful in monitoring biological processes in living cells owing to their practicality and high sensitivity. ${ }^{11,12}$ In recent years, fluorescently labeled probes of bioactive small molecules have become powerful tools to explore biological phenomena and provide insights into the mechanism of actions of these small molecules. ${ }^{13-16}$ With the help of fluorescently labeled probes, this approach makes it applicable in imaging of drug uptake and subcellular distribution. ${ }^{17}$ Besides, it can make the drug-target complex visible by using in-cell imaging, or in-gel

Received: July 16, 2018

Accepted: September 25, 2018

Published: September 25, 2018 
Scheme 1. Synthesis of $N, N$-Diethyl-7-aminocoumarin Derivatives

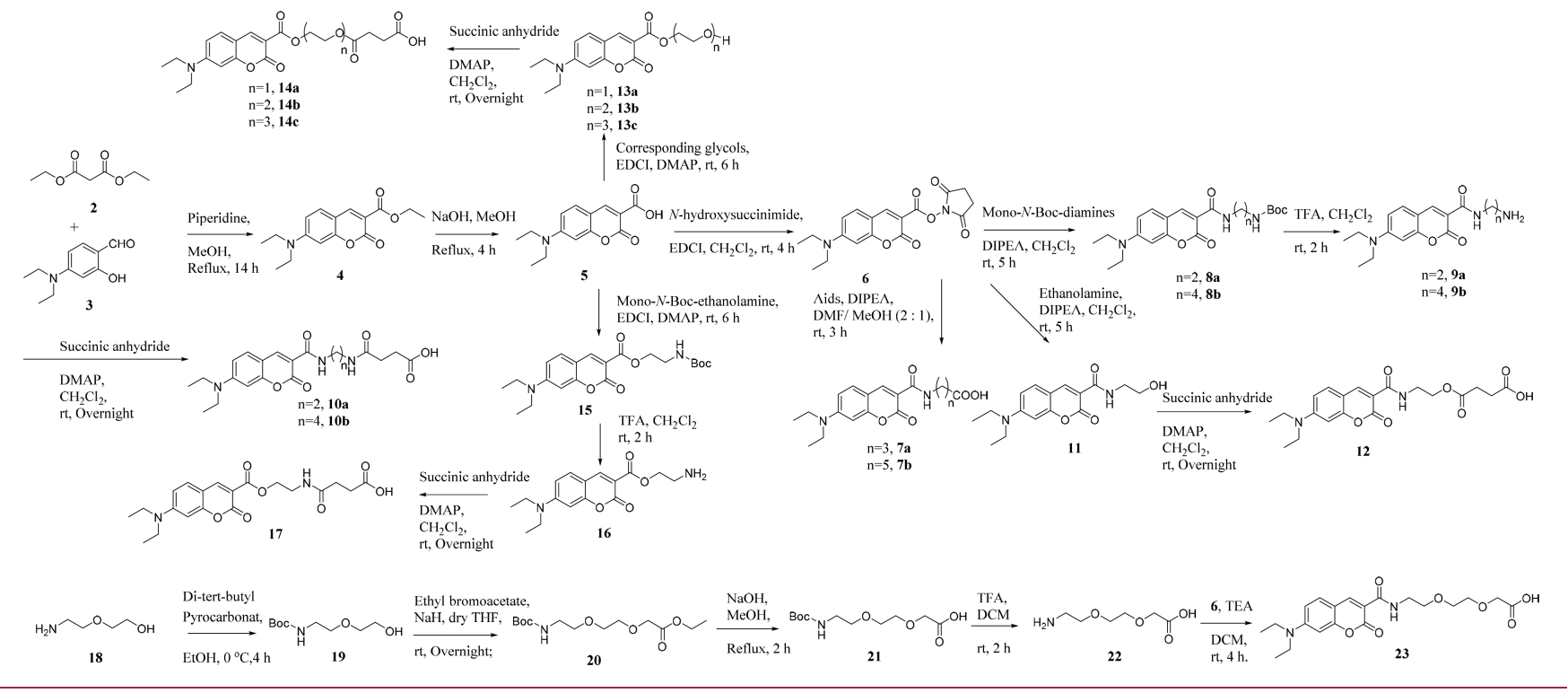

fluorescence scanning after the sodium dodecyl sulfatepolyacrylamide gel electrophoresis (SDS-PAGE) separation. ${ }^{18}$

In our previous studies, we have prepared hundreds of derivatives of 23-HBA to develop novel potential anticancer therapeutics. ${ }^{3-6}$ However, the detailed anticancer mechanism, mode of action, and intracellular target of 23-HBA remain elusive. To better understand the biological mechanism and illuminate the mode of action of 23-HBA, Soural et al. employed a solid-phase synthetic approach to prepare biotinylated 23-HBA; ${ }^{19}$ however, no fluorescent 23-HBA probes have been reported so far to the best of our knowledge.

As our interest continues for developing novel fluorescent probes of natural products for mechanism of action studies, ${ }^{14}$ we herein report the synthesis, biological activities, and labeling performance of fluorescent 23-HBA probes. We expect that the novel fluorescent probes could provide an insight into understanding the subcellular localization and anticancer mechanism of 23-HBA and ultimately accelerate its development as an antitumor agent.

Various conjugation strategies have been developed to construct fluorescent probes of natural products, and the most practical method is attaching a small-molecule fluorescent probe to the tolerated position of natural products with appropriate linkers. ${ }^{20,21}$ In this work, coumarin was selected as the fluorescent probe due to its water-solubility, small size, photostability, and nontoxicity. ${ }^{22,23}$ Various linkers were employed because linker type and length may often affect the antiproliferative activities of probes and their interactions with target proteins. And above all, fluorescent probes must be attached to the compounds at a position that does not interfere with target protein binding. ${ }^{24}$ We recently synthesized hundreds of derivatives of 23-HBA and found that the C-23 hydroxy on 23-HBA can be conjugated with a bulky group without affecting its ability to inhibit cancer cell growth and therefore could be used to attach fluorescent probes.

Fluorescent $N, N$-diethyl-7-aminocoumarin dyes were synthesized according to the route depicted in Scheme 1. Briefly, 4-(diethylamino)salicylaldehyde was reacted with diethyl malonate in the presence of catalytic piperidine to yield coumarin-3-carboxylic acid ethyl ester (4). The ester was then hydrolyzed with sodium hydroxide to obtain acid 5 .
Compound 6 was prepared by the reaction of 5 with $\mathrm{N}$ hydroxysuccinimide in $\mathrm{CH}_{2} \mathrm{Cl}_{2}$. Derivatives $7 \mathbf{a}-\mathbf{b}$ were synthesized by the reaction of compound 6 with corresponding amino acids while $\mathbf{8} \mathbf{a}-\mathbf{b}$ were prepared from the corresponding mono- $N$-boc-diamines. Following deprotection of amino group with trifluoroacetic acid afforded $\mathbf{9 a - b}$. Treated with succinic anhydride in the presence of DMAP, compounds $9 a-b$ were converted to acids $10 \mathbf{a}-\mathbf{b}$. On the other hand, compound 5 afforded alcohols $13 a-c$ upon reaction with corresponding glycols, which were reacted with succinic anhydride to yield subsequent compounds $14 a-c$.

Compounds 12 and 17 were synthesized by reacting with corresponding ethanolamines following the steps described above. In addition, in order to understand the impact of watersoluble ethylene glycol linkers on antiproliferative activities of target compounds, $\mathbf{2 3}$ was synthesized from diglycolamine.

23-HBA was treated with $\mathrm{BnBr}$ in DMF to yield 28-benzyl23-hydroxybetulinic ester (24, Scheme 2). Compounds $\mathbf{7 a - b}$ were converted to acyl chloride and then reacted with ester 24 using DMF as a catalyst in dry $\mathrm{CH}_{2} \mathrm{Cl}_{2}$ to give compounds 25a-b. Target compounds $26 \mathbf{a}-\mathbf{b}$ were obtained by

Scheme 2. Synthesis of Fluorescent 23-HBA Probes $26 a-j^{a}$
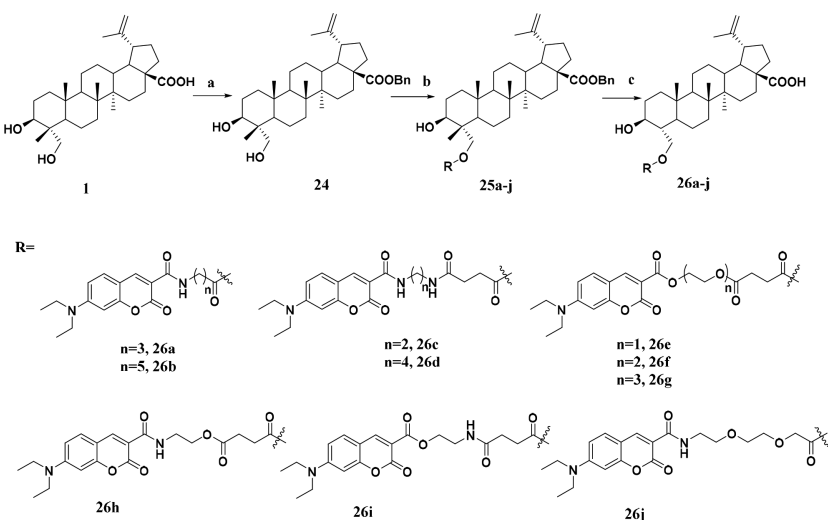

${ }^{a}$ Reagents and conditions: (a) $\mathrm{BnBr}, \mathrm{K}_{2} \mathrm{CO}_{3}, \mathrm{DMF}, \mathrm{rt}, 12 \mathrm{~h}$; (b) Corresponding coumarin acids, oxalyl chloride, triethylamine, cat. DMF, $\mathrm{CH}_{2} \mathrm{Cl}_{2}$, rt, $0.5 \mathrm{~h}$; (c) $\mathrm{H}_{2}, 10 \% \mathrm{Pd} / \mathrm{C}$, THF $/ \mathrm{MeOH}, 50{ }^{\circ} \mathrm{C}, 1 \mathrm{~h}$. 
debenzylation of ester $\mathbf{2 5 a} \mathbf{- b}$ with $10 \% \mathrm{Pd} / \mathrm{C}$ as catalyst under atmospheric pressure of hydrogen. Compounds $26 \mathrm{c}-\mathrm{j}$ were prepared in a route similar to that for $\mathbf{2 6 a}-\mathbf{b}$.

Ideally, the designed natural product fluorescent probes must retain the activity of this natural product. The in vitro antiproliferative activities of newly synthesized fluorescent 23HBA probes were evaluated on two cancer cell lines (B16F10 mice skin melanoma cells, MCF-7 human breast cancer cells) and one normal cell line (Helf human embryonic lung fibroblast cells) by MTT assay. The abilities of these new analogues to inhibit the growth of cells were summarized in Table 1. Most of the derivatives exhibited stronger

Table 1. Antiproliferative Activities of Fluorescent 23-HBA Probes against Different Cell Lines

\begin{tabular}{|c|c|c|c|}
\hline \multirow[b]{2}{*}{ Compounds } & \multicolumn{3}{|c|}{ Cell lines $\left(\mathrm{IC}_{50}, \mu \mathrm{M}\right)^{a}$} \\
\hline & B16F10 & MCF-7 & Helf \\
\hline 23-HBA & $24.46 \pm 2.11$ & $20.83 \pm 1.46$ & $31.49 \pm 1.95$ \\
\hline $26 a$ & $11.27 \pm 0.92$ & $18.06 \pm 1.07$ & $27.76 \pm 0.88$ \\
\hline $26 b$ & $12.27 \pm 0.78$ & $37.77 \pm 1.66$ & $79.65 \pm 3.20$ \\
\hline $26 \mathrm{c}$ & $4.07 \pm 0.05$ & $6.26 \pm 0.27$ & $11.70 \pm 0.85$ \\
\hline $26 d$ & $7.77 \pm 0.33$ & $9.70 \pm 0.49$ & $17.58 \pm 1.21$ \\
\hline $26 \mathrm{e}$ & $11.78 \pm 0.56$ & $19.67 \pm 2.43$ & $23.81 \pm 2.08$ \\
\hline $26 f$ & $35.51 \pm 3.11$ & $30.52 \pm 3.04$ & $21.40 \pm 1.75$ \\
\hline $26 \mathrm{~g}$ & $29.99 \pm 3.44$ & $24.23 \pm 2.51$ & $19.38 \pm 1.38$ \\
\hline $26 \mathrm{~h}$ & $10.53 \pm 0.82$ & $18.13 \pm 1.72$ & $25.12 \pm 4.27$ \\
\hline $26 \mathrm{i}$ & $11.50 \pm 1.81$ & $19.90 \pm 2.06$ & $24.03 \pm 1.53$ \\
\hline $26 j$ & $5.25 \pm 0.93$ & $8.16 \pm 0.12$ & $10.85 \pm 1.05$ \\
\hline $10 \mathrm{a}$ & $>100$ & & \\
\hline $\mathbf{A D M}^{b}$ & $0.19 \pm 0.03$ & $0.15 \pm 0.02$ & $0.21 \pm 0.02$ \\
\hline
\end{tabular}

${ }^{a}$ Each data point represents mean \pm SD from three independent experiments. ${ }^{b} \mathrm{ADM}$ : Doxorubicin.

antiproliferative activities than parent compound 23-HBA against three cell lines, especially on mice skin melanoma B16F10 cells. The antiproliferative activities of $26 \mathrm{a}-\mathbf{i}$ showed that linkers with appropriate length and the introduction of amido groups were favorable to their anticancer activities (Table 1). The most potent derivatives 26c and 26d with lower $\mathrm{IC}_{50}$ values on two tested tumor cell lines had diamine linkers while compounds $\mathbf{2 6} \mathbf{e}-\mathbf{g}$ with ester linkers only had moderate antiproliferative activities ranging from 11.78 to $35.52 \mu \mathrm{M}$. When compared with $26 \mathbf{a}-\mathbf{b}$, enhanced activity of 26j indicated that the introduction of water-soluble ethylene glycol linkers was beneficial to its antiproliferative activity. It should be noted that coumarin dye (10a) exhibited no obvious toxicity against cancer cell lines. Given the potent antiproliferative activity of compound $\mathbf{2 6 c}$, it was finally selected as the candidate for further studies.

The fluorescent properties of compound 26c including the electronic excitation and emission spectra were evaluated first. The results showed that probe $26 \mathrm{c}$ exhibited significant differences between maximum excitation $\left(\lambda_{\text {exc }} 415 \mathrm{~nm}\right)$ and emission $\left(\lambda_{\mathrm{em}} 475 \mathrm{~nm}\right)$ wavelength, namely Stokes shift, which was suitable for the imaging studies (Supporting Information, Figure S1). Then, the cell labeling property of the probe $26 \mathrm{c}$ was investigated by incubating B16F10 cells with various concentrations of $\mathbf{2 6 c}$ in a time dependent manner. The results showed that probe $26 \mathrm{c}$ was taken up into B16F10 cells quickly, and distinguishable fluorescence could be detected as early as 15 min after incubation with $10 \mu \mathrm{M} \mathrm{26c}$ (Figure S2-5). To understand the detailed cellular uptake profile of compound 26c in live B16F10 cells, quantitative research was carried out using flow cytometry. The results showed that after incubation with $10 \mu \mathrm{M} \mathrm{26c}$, the fluorescent intensity increased quickly at the beginning $(0-15 \mathrm{~min})$. Then, a slow growth in fluorescent intensity was observed during the following period of time (15-120 min). Subsequently, the fluorescent intensity grew rapidly (Figure S6). Interestingly, cells stained with naked coumarin dye (10a) were used as a control and the results showed that no obvious fluorescence could be observed after 1 $\mathrm{h}$ staining with up to $50 \mu \mathrm{M}$ of 10a (Figure S7). Considering the poor cell permeability and cytotoxicity of naked coumarin dye $(10 a)$, the above results implied the good cell permeability of 23 -HBA.

The search for cellular targets may well be regarded as a quest for a needle in a giant haystack; the traditional affinitybased proteomics ("pulldown") method often suffers from nonspecific binders. ${ }^{25}$ To minimize unspecific binding in affinity-based approaches, lysate prefractionation may be considered. Use of a particular subcellular fraction will reduce the sample complexity and enrich the target protein. A prerequisite to the fractionation is the knowledge about subcellular localization of the binding proteins which often can be obtained through colocalization study using fluorescently labeled compound. To understand the subcellular localization of $23-\mathrm{HBA}$ in living cells, probe $26 \mathrm{c}$ and commercially available MitoTracker (Mitochondria specific dye) were employed for colocalization studies first. Taking into account the result of qualitative and quantitative research on cellular uptake, the minimum concentration of compound $26 \mathrm{c}$ was optimized to $4 \mu \mathrm{M}$ and the most appropriate incubation time was $1 \mathrm{~h}$. Cells were imaged with confocal laser scanning microscopy. The location of probe $26 \mathrm{c}$ was viewed as green fluorescent spots upon excitation at $415 \mathrm{~nm}$ (Figure 2, left column, green), and the MitoTracker emitted red fluorescence at $579 \mathrm{~nm}$ (Figure 2, right column, red). Overlapping the fluorescent images of probe 26c and MitoTracker showed an extensive orange image in the same area (Figure 2, middle column, orange), revealing the colocalization of these two probes. This observation provided compelling evidence for the

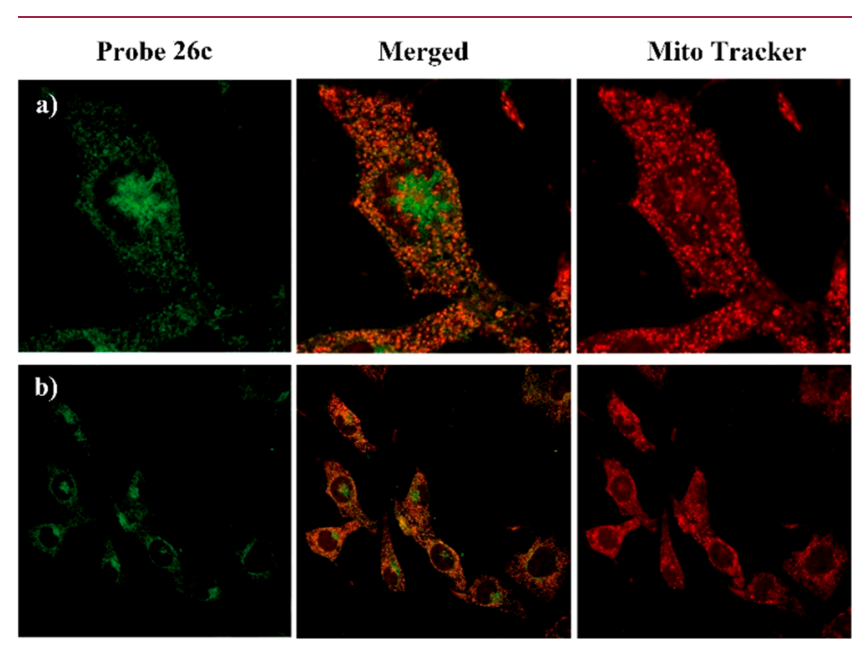

Figure 2. Subcellular colocalization of probe 26c with MitoTracker (mitochondria specific dye) in B16F10 cells. Images were collected using confocal microscopy with excitation of probe 26c at $\lambda_{\mathrm{ex}}=415$ $\mathrm{nm}$ and emission at $\lambda_{\mathrm{em}}=475 \mathrm{~nm}$, MitroTracker at $\lambda_{\mathrm{ex}}=579 \mathrm{~nm}$ and emission at $\lambda_{\mathrm{em}}=600 \mathrm{~nm}$. Images are provided with amplification factor (a) enlarged view of representative cells, 60×, (b) 40×. 
conclusion that mitochondrion is the main accumulation site of 23-HBA, which was consistent with reports revealing that 23-HBA's antitumor mechanism is partially involved in effecting the mitochondrial pathway. On the other hand, some nonmitochondrial regions in cells (near the nucleus) where $26 \mathrm{c}$ is also shown to accumulate are observed in Figure $2 \mathrm{a}$, indicating the possibility of other proteins targeted by 23 HBA. To explain the specificity of the probe for mitochondria, comparative studies with lysosome and nucleus specific dyes are performed and results are shown in Figure 3. Overlapping

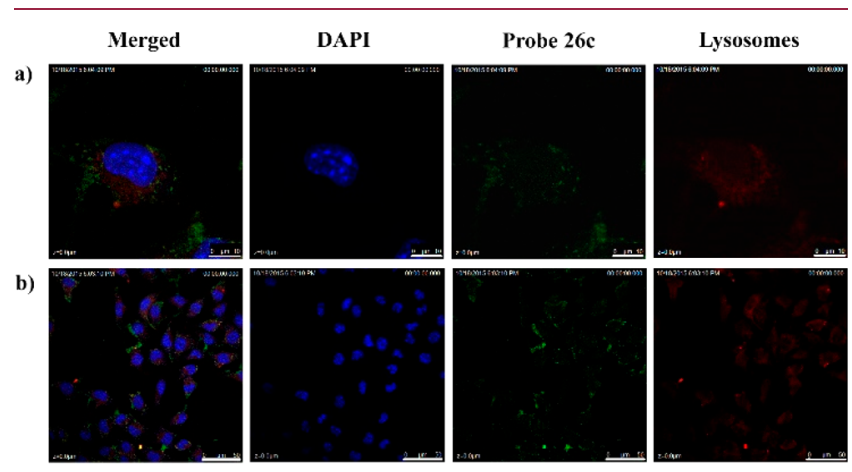

Figure 3. B16F10 cells were costained with 26c $(4 \mu \mathrm{M})$ and LysoTracker Red and then analyzed under a confocal microscope. Nuclei were stained with DAPI. Blue: Nucleus; red: LysoTracker Red,; green: 26c. Micron bar: (a) $10 \mu \mathrm{m}$; (b) $50 \mu \mathrm{m}$.

of the fluorescent images arising from 26c and LysoTracker Red (lysosome specific dye) or DAPI (nucleus specific dye) did not show obvious colocalization. As a result, fluorescent 23-HBA probes provided direct visual evidence that 23-HBA colocalized mainly with mitochondrion in a living cell system and following target identification performed with mitochondrion should be a priority.

Mitochondrion plays an essential role in the intrinsic pathway of apoptosis. Thus, a commercially available fluorescent probe JC-1 was used to measure the MMP of B16F10 cells affected by 23-HBA and 26c using flow cytometry. As shown in Figure 4, B16F10 cells were incubated

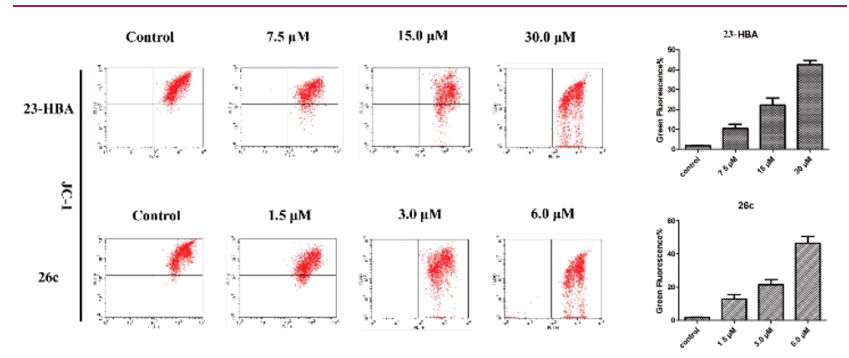

Figure 4. Effect of compounds 23-HBA and 26c on the mitochondrial membrane potentials of B16F10 cells. Cells were incubated with the indicated concentrations of compounds for $48 \mathrm{~h}$ prior to staining with JC-1.

with different concentrations of 23-HBA (0, 7.5, 15, and 30 $\mu \mathrm{M})$ for $48 \mathrm{~h}$ prior to staining with the JC-1. The number of cells with collapsed MMPs in different groups were determined by flow cytometry analysis, yielding $1.79 \%, 10.57 \%, 22.85 \%$, and $42.87 \%$ apoptotic cells, respectively. Similar decreased MMPs were also observed on B16F10 cells treated with $0,1.5$, 3.0, and $6.0 \mu \mathrm{M} \mathrm{26c}$, this result suggests that fluorescent conjugated 23-HBA intracellularly behaves similarly as the starting material 23-HBA.

The above results showed that 23-HBA-induced apoptosis was closely related to the mitochondrial pathway. The Bcl-2 family including pro-apoptotic proteins (e.g., Bax, Bad) and antiapoptotic proteins (e.g., Bcl-2, Bcl-xL) has been identified as essential proteins in controlling the mitochondrial pathway. To confirm whether these proteins are involved in apoptosis induced by 23-HBA, the expression of Bax, Bcl-2, cytochrome c, caspase 3, and caspase 9 was examined by Western Blot analysis. As shown in Figure 5, the expression of Bax,

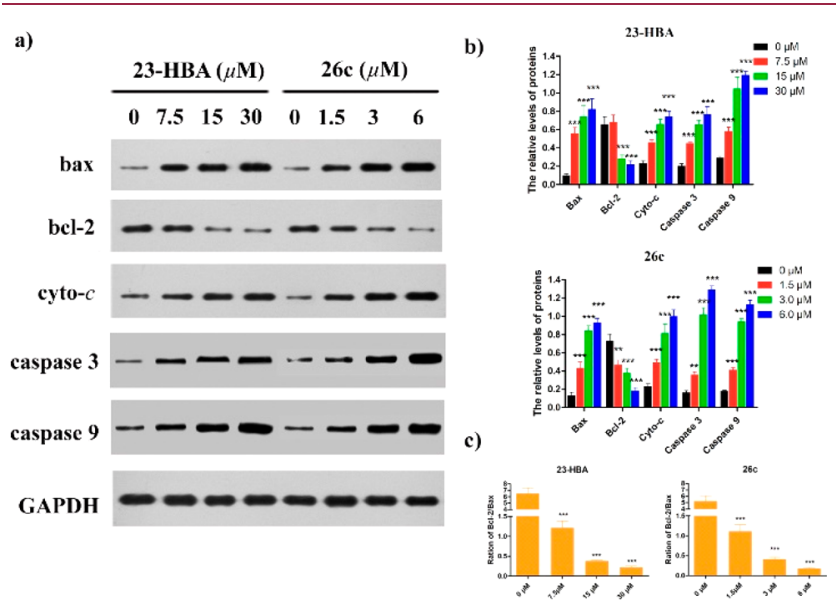

Figure 5. Western Blotting analysis of compounds 23-HBA and 26c on the expression of apoptosis-related proteins bax, bcl-2, cytochrome c, caspase 3, and caspase 9. GAPDH was used as the loading control. (a) B16F10 cells were treated with 23-HBA of various concentrations $(0,7.5,15$, and $30 \mu \mathrm{M})$ and $26 \mathrm{c}(0,1.5,3.0$, and $6.0 \mu \mathrm{M})$. (b) The density ratio of proteins to GAPDH was shown as relative expression, data was represented as mean $\pm \mathrm{SD}$ of three independent experiments. (c) The density ratio between Bcl-2 and Bax. $* * P<$ $0.01, * * * P<0.001$, control compared with treated cells.

cytochrome $c$, caspase 3 , and caspase 9 began to increase after the treatment of $23-\mathrm{HBA}$ or $26 \mathrm{c}$ for $48 \mathrm{~h}$, while the Bcl-2 expression decreased markedly. The above results suggested that the mitochondrial death pathway was involved in 23HBA-induced apoptosis.

In summary, in order to reveal the mechanism of anticancer action of 23-HBA, a series of fluorescent probes of 23-HBA were designed and synthesized by connecting the 23-hydroxyl group of 23-HBA with coumarin dye through various linkers. The pharmacological activity, uptake profile, and subcellular localization of fluorescent 23-HBA probes were investigated in living cells. Qualitative and quantitative research on cellular uptake indicated that probe $26 \mathrm{c}$ was rapidly taken up by B16F10 cells in a dose-dependent manner. Importantly, colocalization studies with organelle specific dyes revealed that mitochondrion is the main site of its accumulation, and subsequent mode of action studies implied that the mitochondrial pathway was involved in the 23-HBA-mediated apoptosis. Overall, our findings indicate that the novel fluorescent probes of 23-HBA could be used for live cell imaging and further to elucidate the mechanisms of anticancer action of 23-HBA. 


\section{ASSOCIATED CONTENT}

\section{S Supporting Information}

The Supporting Information is available free of charge on the ACS Publications website at DOI: 10.1021/acsmedchemlett.8b00321.

Experimental protocols and NMR spectra. (PDF)

\section{AUTHOR INFORMATION}

\section{Corresponding Authors}

*Tel.: +86-25-83271299, E-mail address: jinyixu@china.com (J. $\mathrm{Xu})$.

*Tel.: +86-25-83271299, E-mail address: cpuxst@cpu.edu.cn (S. Xu).

ORCID $\odot$

Wencai Ye: 0000-0002-2810-1001

Jinyi Xu: 0000-0002-1961-0402

\section{Notes}

The authors declare no competing financial interest.

\section{ACKNOWLEDGMENTS}

This work is supported by the National Natural Science Foundation of China (No. 81673306, 81703348) and Newton Fund (No. 201603780078).

\section{ABBREVIATIONS}

23-HBA, 23-hydroxybetulinic acid; MOA, mode of action; SDS-PAGE, sodium dodecyl sulfate-polyacrylamide gel electrophoresis; EDCI, 1-(3-dimethylaminopropyl)-3-ethylcarbodiimide hydrochloride; DMAP, 4-dimethylaminopyridine; DMF, $\mathrm{N}, \mathrm{N}$-dimethylformamide; THF, tetrahydrofuran; MMP, mitochondrial membrane potential; JC-1, 5,5',6,6'-tetrachloro-1,1',3,3'-tetraethyl-imidacarbocyanine iodide

\section{REFERENCES}

(1) Li, G.; Lou, H.-X. Strategies to diversify natural products for drug discovery. Med. Res. Rev. 2018, 38, 1255-1294.

(2) Ye, W.-C.; Ji, N.-N.; Zhao, S.-X.; Liu, J.-H.; Ye, T.; McKervey, M. A.; Stevenson, P. Triterpenoids from Pulsatilla chinensis. Phytochemistry 1996, 42, 799-802.

(3) Zhu, P. Q.; Bi, Y.; Xu, J. Y.; Li, Z.; Liu, J.; Zhang, L. Y.; Ye, W. C.; $\mathrm{Wu}, \mathrm{X}$. M. Terpenoids. III: Synthesis and biological evaluation of 23-hydroxybetulinic acid derivatives as novel inhibitors of glycogen phosphorylase. Bioorg. Med. Chem. Lett. 2009, 19, 6966-6969.

(4) Zhang, H. Y.; Zhu, P. Q.; Liu, J.; Lin, Y.; Yao, H. Q.; Jiang, J. Y.; Ye, W. C.; Wu, X. M.; Xu, J. Y. Synthesis, in vitro and in vivo antitumor activity of pyrazole-fused 23-hydroxybetulinic acid derivatives. Bioorg. Med. Chem. Lett. 2015, 25, 728-732.

(5) Zhang, H. Y.; Wang, Y. W.; Zhu, P. Q.; Liu, J.; Xu, S. T.; Yao, H. Q.; Jiang, J. Y.; Ye, W. C.; Wu, X. M.; Xu, J. Y. Design, synthesis and antitumor activity of triterpenoid pyrazine derivatives from 23hydroxybetulinic acid. Eur. J. Med. Chem. 2015, 97, 235-244.

(6) Zhang, H. Y.; Zhu, P. Q.; Liu, J.; Yang, X.; Xu, S. T.; Yao, H. Q.; Jiang, J. Y.; Ye, W. C.; Wu, X. M.; Xu, J. Y. Synthesis and antitumor activity of novel 3-oxo-23-hydroxybetulinic acid derivatives. Eur. J. Med. Chem. 2014, 87, 159-167.

(7) Liu, M.; Zhao, X. Z.; Xiao, L.; Liu, G.; Liu, H. Z.; Wang, X. Y.; Feng, X.; Lin, X. K. Cytotoxicity of the compounds isolated from Pulsatilla chinensis saponins and apoptosis induced by 23 hydroxybetulinic acid. Pharm. Biol. 2015, 53, 1-9.

(8) Yao, N.; Li, Y. J.; Zhang, D. M.; Liu, D. L.; Tang, M. K.; Yiu, A.; Li, Y.; Chen, W. M.; Lan, P.; Yao, Z.; Chen, Z. S.; Ye, W. C. B4G2 induces mitochondrial apoptosis by the ROS-Mediated opening of $\mathrm{Ca}^{2+}$-dependent permeability transition pores. Cell. Physiol. Biochem. 2015, 37, 838-852.
(9) Pan, S. J.; Zhang, H. L.; Wang, C. Y.; Yao, S. C.; Yao, S. Q. Target identification of natural products and bioactive compounds using affinity-based probes. Nat. Prod. Rep. 2016, 33, 612-620.

(10) Fetz, V.; Prochnow, H.; Brönstrup, M.; Sasse, F. Target identification by image analysis. Nat. Prod. Rep. 2016, 33, 655-667.

(11) Umezawa, K.; Yoshida, M.; Kamiya, M.; Yamasoba, T.; Urano, $\mathrm{Y}$. Rational design of reversible fluorescent probes for live-cell imaging and quantification of fast glutathione dynamics. Nat. Chem. 2017, 9, 279-286.

(12) Liu, Z.; Zhou, X.; Miao, Y.; Hu, Y.; Kwon, N.; Wu, X.; Yoon, J. A. Reversible fluorescent probe for real-time quantitative monitoring of cellular glutathione. Angew. Chem., Int. Ed. 2017, 56, 5812-5816.

(13) DeGuire, S. M.; Earl, D. C.; Du, Y.; Crews, B. A.; Jacobs, A. T.; Ustione, A.; Daniel, C.; Chong, K. M.; Marnett, L. J.; Piston, D. W.; Bachmann, B. O.; Sulikowshi, G. A. Fluorescent probes of the apoptolidins and their utility in cellular localization studies. Angew. Chem., Int. Ed. 2015, 54, 961-964.

(14) Xu, S. T.; Luo, S. S.; Yao, H.; Cai, H.; Miao, X. M.; Wu, F.; Yang, D. H.; Wu, X. M.; Xie, W. J.; Yao, H. Q.; Chen, Z. S.; Xu, J. Y. Probing the anticancer action of oridonin with fluorescent analogues: visualizing subcellular localization to mitochondria. J. Med. Chem. 2016, 59, 5022-5034.

(15) Xu, W.; Zeng, Z.; Jiang, J.-H.; Chang, Y.-T.; Yuan, L. Discerning the chemistry in individual organelles with small-molecule fluorescent probes. Angew. Chem., Int. Ed. 2016, 55, 13658-13699.

(16) Zhang, X.; Ba, Q.; Gu, Z. N.; Guo, D. L.; Zhou, Y.; Xu, Y. G.; Wang, H.; Ye, D. J.; Liu, H. Fluorescent coumarin-artemisinin conjugates as mitochondria-targeting theranostic probes for enhanced anticancer activities. Chem. - Eur. J. 2015, 21, 17415-17421.

(17) Wu, J.; Shen, Q.; Wang, Y.; Zhao, D.; Peng, C.; Li, J.-X. Fluorescent probes for subcellular localization during osteoclast formation. ACS Med. Chem. Lett. 2014, 5, 911-914.

(18) Takahashi, M.; Kawamura, A.; Kato, N.; Nishi, T.; Hamachi, I.; Ohkanda, J. Phosphopeptide-dependent labeling of 14-3-3 $\zeta$ proteins by fusicoccin-based fluorescent probes. Angew. Chem., Int. Ed. 2012, $51,509-512$.

(19) Soural, M.; Hodon, J.; Dickinson, N. J.; Sidova, V.; Gurska, S.; Dzubak, P.; Hajduch, M.; Sarek, J.; Urban, M. Preparation of conjugates of cytotoxic lupane triterpenes with biotin. Bioconjugate Chem. 2015, 26, 2563-2570.

(20) Jurášek, M.; Rimpelová, S.; Kmoníčková, E.; Drašar, P.; Ruml, $\mathrm{T}$. Tailor-made fluorescent trilobolide to study its biological relevance. J. Med. Chem. 2014, 57, 7947-7954.

(21) Pal, A.; Ganguly, A.; Chowdhuri, S.; Yousuf, M.; Ghosh, A.; Barui, A. K.; Kotcherlakota, R.; Adhikari, S.; Banerjee, R. Bis-arylidene oxindole-betulinic acid conjugate: a fluorescent cancer cell detector with potent anticancer activity. ACS Med. Chem. Lett. 2015, 6, 612616.

(22) Hori, Y.; Norinobu, T.; Sato, M.; Arita, K.; Shirakawa, M.; Kikuchi, K. Development of fluorogenic probes for quick no-wash live-cell imaging of intracellular proteins. J. Am. Chem. Soc. 2013, 135, $12360-12365$.

(23) Rong, L.; Liu, L. H.; Chen, S.; Cheng, H.; Chen, C. S.; Li, Z. Y.; Qin, S. Y.; Zhang, X. Z. A coumarin derivative as a fluorogenic glycoproteomic probe for biological imaging. Chem. Commun. 2014, 50, 667-669.

(24) Kanoh, N. Photo-cross-linked small-molecule affinity martix as a tool for target identification of bioactive small molecules. Nat. Prod. Rep. 2016, 33, 709-718.

(25) Ziegler, S.; Pries, V.; Hedberg, C.; Waldmann, H. Target identification for small bioactive molecules finding the needle in the haystack. Angew. Chem., Int. Ed. 2013, 52, 2744-2792. 